\title{
Journal of Nutritional Disorders and
}

\section{Difficult Postoperative Extubation in a Patient with Hypoproteinemia: A Case Report}

\author{
Peilin Cong, Hao Zhang, Yilu Zhou, Bi Xia and Qingxiu Wang*
}

Department of Anesthesiology, Shanghai East Hospital, Tongji University School of Medicine, PR China

*Corresponding author: Qingxiu Wang Ph.D, M.D, Department of Anesthesiology, Shanghai East Hospital, Tongji University School of Medicine, 150 Jimo Road, Shanghai 200120, PR China, Tel: +1-500-079-0452; E-mail: qxw1123@126.com

Received date: October 30, 2016; Accepted date: February 20, 2017; Published date: February 27, 2017

Copyright: () 2017 Cong P, et al. This is an open-access article distributed under the terms of the Creative Commons Attribution License, which permits unrestricted use, distribution, and reproduction in any medium, provided the original author and source are credited.

\begin{abstract}
Hypoalbuminemia is common in critically ill patients, chronic hypoproteinemia tend to cause some negative impacts on the body's internal environment. Hypoalbuminemia poses many challenges to the perioperative anesthesia management. This article describes a case of difficult postoperative extubation in a 62-year-old woman with hypoalbuminemia. She was diagnosed with intestinal tuberculosis, hypoproteinemia and edema for 3 years. During the anesthesia management process, we found some physiological changes in hypoproteinemia and the management of anesthesia during perioperative period is complex. So in this article, we provide some advice for the treatment and anaesthetic management of patients with hypoproteinemia.
\end{abstract}

Keywords: Hypoproteinemia; Pulmonary edema; Extubation difficult; Albumin management

\section{Introduction}

Hypoalbuminemia is common in critically ill patients; it generally defined as a serum albumin concentration $\leq 30 \mathrm{~g} / \mathrm{l}$ [1]. Albumin reduction is more common in albumin synthesis abnormalities caused by liver function abnormalities or loss of serum albumin loss too much caused by vomiting diarrhea. Moreover, it is well known that albumin has many important physiological functions, it is the most important factor to maintain the plasma colloid osmotic pressure and also affect the permeability of capillaries, which may cause a redistribution of body fluid; changes of plasma albumin concentration will affect the normal homeostasis as well as the delivery and effective of drugs; scavenging oxygen free radicals; inhibition of platelet function to antithrombotic $[1,2]$. Albumin is the maximum protein in human plasma with remarkably diverse functions including antioxidant activity, buffering properties, binding and transport capacities for numerous substances (free fatty acids, ions, NO, bilirubin, peptides, uremic toxins and drugs)[3]. So before the full treatment, low protein levels always bring great challenges to the surgeries and anesthesia. It is important to avoid inappropriate treatment and improve prognosis. This article describes a case of an unexpected decrease in blood oxygen saturation in patients with hypoproteinemia after operation, and provides some suggestions for the management of patients with hypoproteinemia.

\section{Case Report}

A 62 year old female with a weight of $48 \mathrm{~kg}$ and a height of $154 \mathrm{~cm}$ was admitted to the hospital because of the emergence of diarrhea and the edema. She was diagnosed as intestinal tuberculosis with abdominal lymph node tuberculosis (T-SPOT (A antigen) 20; T-SPOT (B antigen) 8) 3 years ago and received the antituberculosis therapy treatment (HRE) for 18 months. But after stopping the drug, the diarrhea still occurred for many times and accompany with the complication of hypoproteinemia associated with edema. The patient was received anti-diarrhea treatment and artificial albumin supplementation; however no obvious remission in the patient's condition was exhibited. Her Plasma albumin concentration was fluctuated between $20 \mathrm{~g} / \mathrm{L}$ and $31 \mathrm{~g} / \mathrm{L}$ during 3 years.

The latest enteroscopy examination (Figure 1) showed duodenum polyp \& ileocecal junction polyp. So she was planned to perform an operation of intestinal polyps' excision under general anesthesia. Before the surgery, edema could be found in her arms, legs and eyelids. Laboratory examination of plasma albumin for $23 \mathrm{~g} / \mathrm{L}$, the test result showed normal chest radiography, normal ECG, hemoglobin $10 \mathrm{~g} / \mathrm{dl}$. For general anesthesia induction, $20 \mu \mathrm{g}$ sufentanil, $100 \mathrm{mg}$ propofol, 40 $\mathrm{mg}$ rocuronium for the endotracheal intubation were intravenously injected. Tracheal intubation was successfully conducted with a single lumen tube of ID.6.5\# for depth $21 \mathrm{~cm}$. The operation was smooth and the vital signs were all stable throughout the surgery (systolic/diastolic blood pressure $110-140 / 60-80 \mathrm{mmHg}$, heart rate $69-80$ beats/min and pulse oxygen saturation 100\%). The injected fluid included crystalloid (Sodium lactate) $1000 \mathrm{ml}$. After the finish of operation, $1 \mathrm{mg}$ neostigmine and $0.5 \mathrm{mg}$ atropine were given as the antagonist of muscle relaxant. To observe the patient's breathing, manual control was given when the patient is conscious. However, the heart rate was rapidly increased to $118 / \mathrm{min}$, peak airway pressure $40 \mathrm{cmH} 2 \mathrm{O}$ and the ETCO2 was $55 \mathrm{mmHg}$. The oxygen saturation continued to decline to $85 \%$ and the blood pressure was $90 / 58 \mathrm{mmHg}$. During this series of operations, abundant airway secreta was found and Coarse inspiratory rhonchi were heard on auscultation.

In that case, a possibility of pulmonary edema which caused by chronic low serum albumin was considered. So the patient was given mechanical ventilation with PEEP $5 \mathrm{cmH} 2 \mathrm{O}$ ventilation and the aminophylline $0.02 \mathrm{~g}$ immediately. After these treatments, the oxygen saturation has rebounded to $87-88 \%$, however her vital signs were not improved. After that, the patient received a methylprednisolone $40 \mathrm{mg}$, furosemide $10 \mathrm{mg}$ injection and inhalant salbutamol, semireclining position was taken to assist her respiration. After taking these measures, the blood oxygen saturation gradually recovered to $95 \%$. 


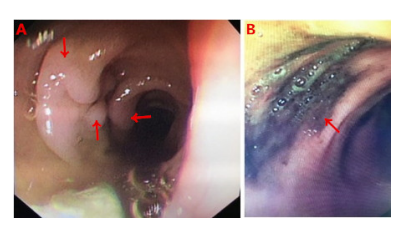

Figure1: The enteroscopy examination shows that the patient has polypus and ulceration in ileocecal junction and colon. A: Dedicate edema of intestinal wall and a lot of polypus. B: Shows ulceration and inflammatory effusion in the colon.

After vital signs of the patient maintain at a stable level, endotracheal tube was extubated. The patient was then transferred to PACU for a further observation.

\section{Discussion}

Hypoalbuminemia was defined as serum albumin levels less than 30 g/l. Hypoalbuminemia can cause complications increase poor prognosis. In a meta-analysis of 90 cohort studies that had evaluated hypoalbuminemia as a prognostic biomarker in acutely ill patients, each $10 \mathrm{~g} / \mathrm{l}$ decrease in serum albumin concentration was associated with a $137 \%$ increase in the odds of death, $89 \%$ increase in morbidity and $71 \%$ increase in length of hospital stay [1]. Hypoalbuminemia always is the complication of some diseases, so lay too much stress on primary diseases may neglect the negative influences caused by hypoalbuminemia.

Maintain the colloid osmotic pressure (COP) is considered albumin's major function as it controls the distribution of extracellular fluid between the vascular and extra-vascular compartments [3]. Starling formula describes the factors that control the liquid filter by the capillary wall. Explains the normal fluid of lung continue to filter and keep the lungs moist and also expounds two kinds of the mechanism of pulmonary edema (cardiac and non-cardiac).

$$
Q f=K f[(P m v-P i)-\sigma(\pi m v-\pi i)]
$$

Kf: coefficient of capillary permeability; Pmv: the capillary lumen hydrostatic pressure; Pi: the alveolar interstitial hydrostatic pressure; $\sigma$ : reflection coefficient (the effectiveness of the vascular barrier in preventing diffusion of protein); $\pi \mathrm{mv}$ : the microvascular protein osmotic pressure; $\pi \mathrm{i}$ : the interstitial protein osmotic pressure [4].

The Pmv, $\pi m v$ and capillary permeability are critical factors which control the liquid volume filter from blood capillary. When hypoproteinemia reduces the interstitial protein osmotic pressure $(\pi i)$, the microvascular protein osmotic $(\pi \mathrm{mv})$ pressure will decrease too, so pulmonary edema is rarely met in hypoproteinemia. But when accompanied by the increasing of the capillary lumen hydrostatic pressure (Pmv) in microvascular and capillary permeability changes, it is easy to induce pulmonary edema [5].

As for this case, when removing the endotracheal tube, there were obvious clinical manifestations of pulmonary edema, vital signs changed, symptoms the moist rale in the lungs and bubble sputum. Therefore, we consider the possibility of pulmonary edema which caused by chronic hypoalbuminemia and made the appropriate treatment. Acute pulmonary edema is one of the most serious complications occurred during perioperative period. If treatment is not timely when acute pulmonary edema happened, patients may be dying from cardiorespiratory failure. For this patient, before the anesthesia edema caused by the change of COP exist not only in arms and legs, but also in the tissue of body. Infuse a large number of crystal liquid Intraoperative will dilute the plasma albumin, this will decrease $\mathrm{COP}$ and increase capillary lumen hydrostatic pressure (Pmv). In this time, it is easy to induce the formation of edema fluid according to the starling formula. Moreover, the acute pulmonary edema during the perioperative often associated with a number of predisposing factors such as remove the IPPV, increase of cardiac output caused by over fluid infusion during the operation, Airway obstruction, high blood pressure[6]. Extubation and sputum suction are both high risk factors to induce the acute pulmonary edema. When using the positive pressure mechanical ventilation can prevent blood flow to the pulmonary interstitial, these maybe hide the fluid that accumulates in the pulmonary interstitial [7].

The early clinical manifestations of pulmonary edema are cough and chest tightness, dyspnea, moist rale can be found in lungs. The cardiac output, blood pressure and $\mathrm{PaO}_{2}$ will all decrease. Use arterial blood gas analysis to analysis is an effective way to make an early diagnosis Chest X-ray can diagnose pulmonary edema, but its accuracy is limited. Edema may not be visible until the amount of lung water increases by 30 percent. Pulmonary-artery catheterization is considered the gold standard for determining the cause of acute pulmonary edema.

The case of perioperative pulmonary edema is not uncommon, but the example of pulmonary edema induced by hypoalbuminemia is uncommon, but the management of chronic hypoalbuminemia patients should be paid more attention. Hypoalbuminemia has been shown to be associated with increased mortality and morbidity rates in both hospitalized patients in surgery, an association between hypoalbuminemia and adverse outcome has been recognized for many years. Hypoalbuminemia significantly influences specifically surgical site infection and DVT formation. Surgical delay for preoperative nutrition has been shown to improve the morbidity and mortality in patients with severe nutritional risk [2-8].

Before operation the test of plasma albumin concentration and colloid osmotic pressure are both helpful to estimate the edema of tissue. So, improving hypoproteinemia together with weight gain by increase of protein and calorie intakes and periodic nutrition management by using protein supplements in the patient of proteinlosing enteropathy with edema and hypoproteinemia would be a meaningful precedent [9]. Albumin administration can result in lower fluid requirements and reduced pulmonary and intestinal oedema compared with compared with other fluid regimens (crystalloids) [10]. A dose of frusemide administered will prevent rebound hypervolaemia and pulmonary oedema after the fluid resuscitation, this will mobilise large quantities of odema as urine, re-establish a stable circulation, and will have a virtually guaranteed survival [11]. A low concentration of plasma albumin will increase the dissociative anaesthetic, so delayed recovery are likely to occur. Ting Zhang et al. [12] showed that dexmedetomidine infusion can safely be used in mild hypoalbuminemia patients after major abdominal or thoracic surgery. The patients with no contraindications can choose small incision epidural anesthesia; epidural anesthesia is generally regarded as to be safe in many surgical operations. At last, a smooth extubation process can reduce stimulation is more advantageous to reduce the possibility of pulmonary edema. 
Citation: Cong P, Zhang H, Zhou Y, Xia B, Wang Q (2017) Difficult Postoperative Extubation in a Patient with Hypoproteinemia: A Case Report. J Nutr Disorders Ther 7: 207. doi:10.4172/2161-0509.1000207

Page 3 of 3

\section{Disclosure}

Written informed consent was obtained from the patient upon discharge for publication of this manuscript. The authors report no conflict of interest in this work

\section{References}

1. Jean-Louis Vincent, James A Russell, Matthias Jacob, Greg Martin, Bertrand Guidet, et al. (2014) Albumin administration in the acutely ill: what is new and where next? Crit Care18: 231.

2. Truong A, Hanna MH (2016) Implications of preoperative hypoalbuminemia in colorectal surgery. World J Gastrointest Surg 8: 353-362.

3. Michelis R, Sela S, Zeitum T, Geron R, Kristal B (2016) Unexpected Normal Colloid Osmotic Pressure in Clinical States with Low Serum Albumin. PLoS One 11: e0159839.

4. Bhattacharya M, Kalle RH (2016) Negative-Pressure Pulmonary Edema. Chest 150: 927-933.

5. Ware LB, Matthay MA (2005) Acute Pulmonary Edema. N Engl J Med 353: $2788-2796$
6. Butterworth JF, Mackey DC, Wasnick JD. Morgan\&Mikhail's Clinical Anesthesiology, 5th edition, LANGE medical book.

7. Zhen D, Xiu L (2008) Treatment of Cardiac pulmonary edema by noninvasive positive pressure ventilation (NIPPV). Clin Pulm Med 1:13.

8. Gibbs J, Cull W, Henderson W, Daley J, Hur K, et al. (1999) Preoperative Serum Albumin Level as a Predictor of Operative Mortality and Morbidity. Arch Surg 134: 36-42.

9. Hyun Jeong Lee, Mi Yong Rha, Young Yun Cho, Eun Ran Kim, Dong Kyung (2012) A Case of Protein Supplement Effect in Protein-Losing Enteropathy. Clin Nutr Res 1: 94-98.

10. Haynes GR, Navickis RJ, Wilkes MM (2003) Albumin administration: what is the evidence of clinical benefit? A systematic review of randomized controlled trials. Eur J Anaesthesiol 20: 771-793.

11. Coulthard MG (2015) Oedema in kwashiorkor is caused by hypoalbuminaemia. Paediatr Int Child Health 35: 83-89.

12. Zhang T, Deng Y, He P, He Z, Wang X (2015) Effects of mild hypoalbuminemia on the pharmacokinetics and pharmacodynamics of dexmedetomidine in patients after major abdominal or thoracic surgery. J Clin Anesth 27: 632-637. 